\title{
Bone and Tendon Coverage via Dehydrated Human Amniotic/Chorionic Membrane and Split-Thickness Skin Grafting
}

\author{
Radbeh Torabi, MD ${ }^{1}$ Amy L. Strong, $\mathrm{PhD}, \mathrm{MPH}^{2}$ \\ Oren Tessler, MD, MBA ${ }^{1}$ Frank H. Lau, MD ${ }^{1}$ \\ ${ }^{1}$ Division of Plastic and Reconstructive Surgery, Louisiana State \\ University School of Medicine, New Orleans, Louisiana \\ ${ }^{2}$ Center for Stem Cell Research and Regenerative Medicine, Tulane \\ University School of Medicine, New Orleans, Louisiana \\ J Reconstr Microsurg Open 2016;1:59-62.
}

Extremity wounds are debilitating and complex. When critical structures are not exposed, extremity wounds can be managed with conservative measures such as local wound care or skin grafting. ${ }^{1}$ However, exposure of critical structures such as bone or tendon precludes the use of these modalities. Skin grafts do not engraft onto bone and tendon, and with local wound care alone, bone develops osteomyelitis and tendons desiccate and rupture. ${ }^{2}$ Without soft tissue reconstruction, osteomyelitis leads to amputation $50 \%$ of the time and tendon rupture results in loss of function. ${ }^{2}$

Free flap reconstruction is currently the gold standard for limb salvage. The high technical expertise required for consistent free flap limb salvage poses a barrier for patient access to care, and not all patients are suitable candidates. Additionally, microsurgical free flaps in the lower extremity have an $8 \%$ failure rate, $75 \%$ of which proceed to amputation. ${ }^{3}$ Reliable alternatives to free flap reconstruction that lead to stable wound closure are needed.

Dehydrated human amniotic/chorionic membrane (dHACM) is an FDA-approved biologic device that delivers a large number of growth factors. ${ }^{4}$ While dHACM is effective at closing diabetic foot ulcers and venous stasis ulcers, ${ }^{5,6}$ many applications of dHACM over prolonged periods are typically necessary for complete closure. The cost and time to closure makes DHACM alone an unsuitable method for limb salvage.

We developed a novel limb salvage technique using dHACM to generate granulation tissue over critical structures and then definitively closing the wound with splitthickness skin grafts (STSG). Tendon coverage requires
Address for correspondence Frank H. Lau, MD, Division of Plastic and Reconstructive Surgery, Louisiana State University School of Medicine, 1901 Perdido Street, New Orleans, LA 70112 (e-mail: flau@lsuhsc.edu).

1 week of dHACM treatment before STSG. Bone coverage requires 2 to 3 weeks of dHACM treatment before STSG. We present our early experiences using this innovative approach.

\section{Materials and Methods}

dHACM + STSG limb salvage ( - Table $\mathbf{1}$ ) was guided by the following parameters. Following initial debridement, wounds were assessed for size and surface area of exposed bone and/or tendon. dHACM was applied to the wounds to generate granulation tissue, and wounds were covered with a nonadherent dressing. During the ensuing week, wounds were assessed for granulation tissue. If sufficient, STSG was performed. If the granulating base was not ready, additional dHACM application was performed. Wounds were monitored carefully for infection.

\section{Results}

Between November 5, 2014, and March 30, 2015, seven patients underwent dHACM + STSG limb salvage. Demographics included 8 to 64 years of age, and two female and five male patients. Wounds included two with exposed tendons, three with exposed bone, and two with exposed bone and tendon. dHACM and STSG was successful in six of the seven patients. None developed infection during dHACM treatment, STSG, and in the postoperative phase, even in the cases where initial antibiotic treatment was inadequate due to bacterial resistance. All wounds remain stably closed. received

June 22, 2015

accepted

November 28, 2015

published online

January 18, 2016
DOI http://dx.doi.org/

10.1055/s-0036-1571279.

ISSN 2377-0813.
Copyright $\odot 2016$ by Thieme Medical Publishers, Inc., 333 Seventh Avenue, New York, NY 10001, USA.

Tel: +1(212) 584-4662.
License terms

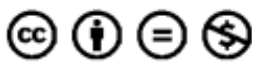


Table 1 Demographics and clinical information of our case series

\begin{tabular}{|l|l|l|l|l|l|l|}
\hline Age $(\mathrm{y})$ & Sex & Wound size $\left(\mathbf{c m}^{2}\right)$ & Location & Critical structure(s) exposed & No. of dHACM & Successful STSG? \\
\hline 8 & F & 16 & LE & Achilles tendon & 1 & Yes \\
\hline 37 & F & 6 & LE & Extensor tendons of foot & 1 & Yes \\
\hline 44 & M & 3 & UE & Pectoral tendon, humerus & 1 & Yes \\
\hline 60 & M & 12 & LE & Tibia & 2 & Yes \\
\hline 64 & M & 8 & LE & Tibia & 2 & Yes \\
\hline 54 & M & 12 & LE & Tibia and tibialis anterior tendon & 2 & Unknown \\
\hline 47 & M & 5 & LE & Tibia and tibialis posterior tendon & 3 & Yes \\
\hline
\end{tabular}

Abbreviations: LE, lower extremity; UE: upper extremity.

\section{Selected Case Reports}

\section{Case I: Foot Extensor Tendons}

A 37-year-old woman whose foot was run over by a garbage truck presented with a right foot dorsum degloving injury with exposed extensor tendons and multiple closed fractures. Comorbidities included uncontrolled type 2 diabetes mellitus. The wound was treated three times with urinary bladder matrix with neither granulation tissue generation nor wound

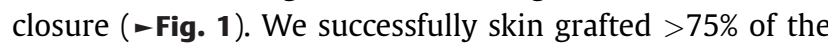
wound but $6 \mathrm{~cm}^{2}$ of exposed extensor tendons remained. One dHACM treatment was applied to the tendons and STSG was successful the following week. The wound remained stably closed at 2-month follow-up and the patient resumed ambulation.

\section{Case II: Tibia with Chronic MRSA Osteomyelitis}

A 64-year-old man presented with more than 20 years history of a draining midtibial wound following a motor vehicle collision. Comorbidities included diabetes, hyperlipidemia, hypertension, and chronic obstructive pulmonary disease. We performed wide soft tissue and bony debridement, resulting in $8 \mathrm{~cm}^{2}$ of exposed tibia, placed $\mathrm{dHACM}$, and started intravenous (IV) cefazolin (-Fig. 2). Three days after dHACM placement, bony cultures grew methicillin-resistant Staphylococcus aureus (MRSA) and switched to IV vancomycin. Notably, no clinical infection developed. Two additional dHACM treatments were applied, adequate granulation tissue developed, and STSG was successful. Of note, the superolateral corner of the wound was not treated with dHACM, resulting in persistently exposed tibia which was closed with
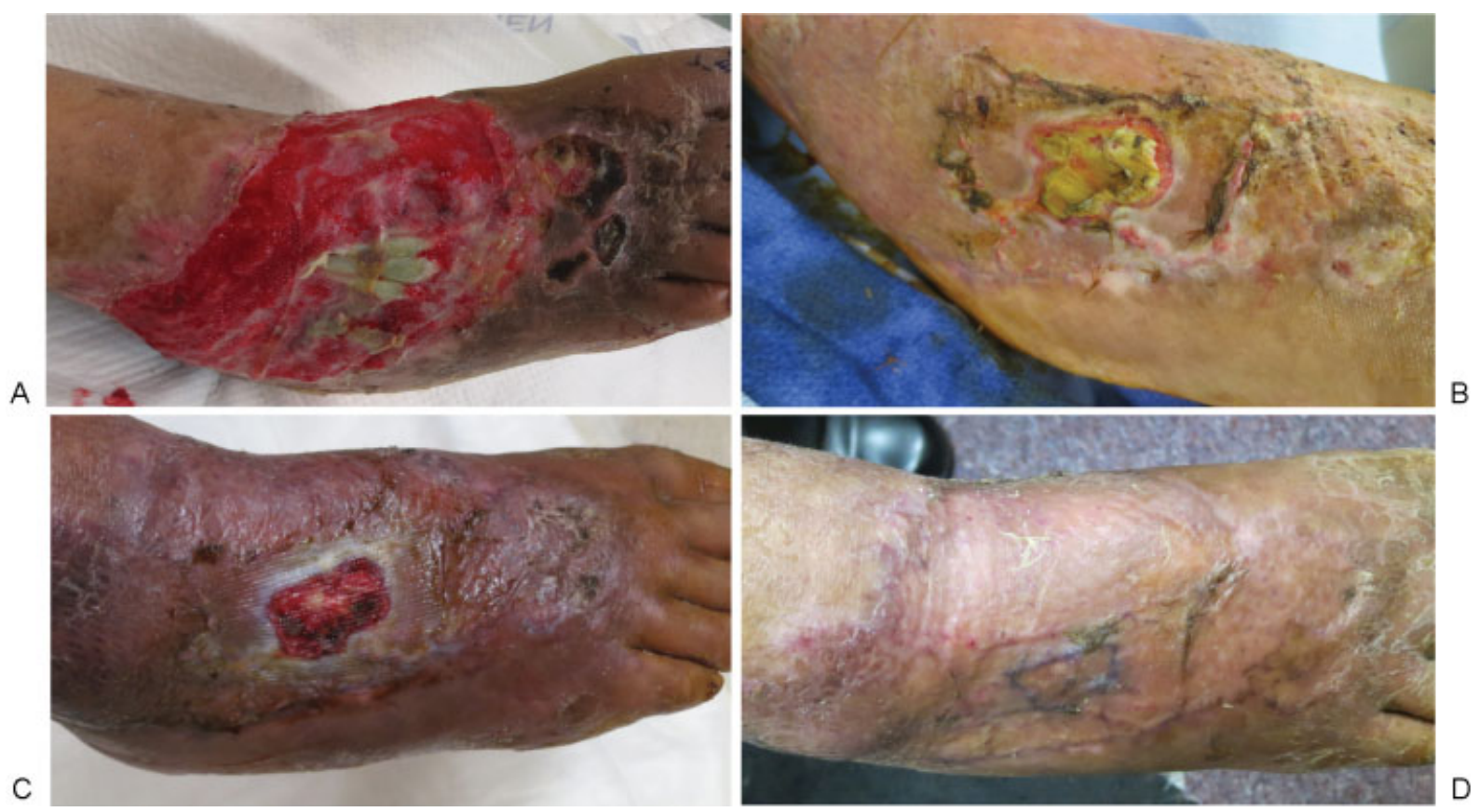

Fig. 1 A 37-year-old woman who was struck by a motor vehicle presented with a degloving injury of her right dorsal foot. (A) On initial referral, the patient had been treated three times with urinary bladder matrix, without coverage of her exposed extensor tendons. (B) Majority of the wound was successfully skin grafted except for central portion with exposed tendon. dHACM was applied once. (C) The wound demonstrated adequate granulation tissue 1 week later and she underwent STSG. (D) Stable closure 4 weeks following STSG. 
A
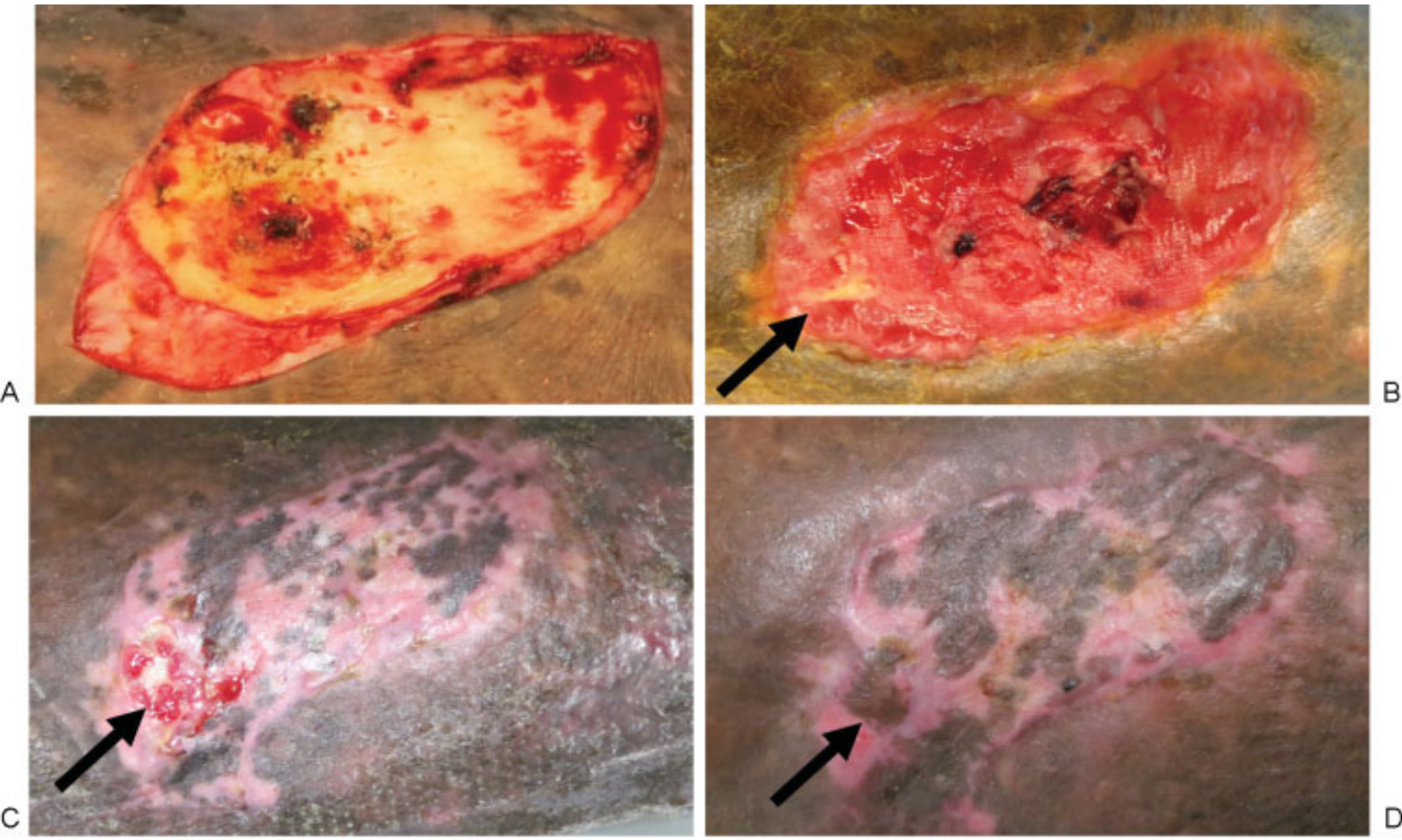

Fig. 2 A 64-year-old man presented with chronic osteomyelitis and exposed tibia of more than 20 years following a motor vehicle collision. (A) Soft tissue and bony debridement was performed and DHACM was applied. (B) Adequate granulation tissue overlying bone after three dHACM applications. Black arrow marks bony region that was not covered by dHACM. (C) STSG was placed on granulation tissue, while a rotational flap was performed to cover the untreated bone (black arrow), demonstrating stable closure 5 weeks postoperatively, while the rotation flap required local wound care. (D) Wound appearance 2 months postSTSG.

A
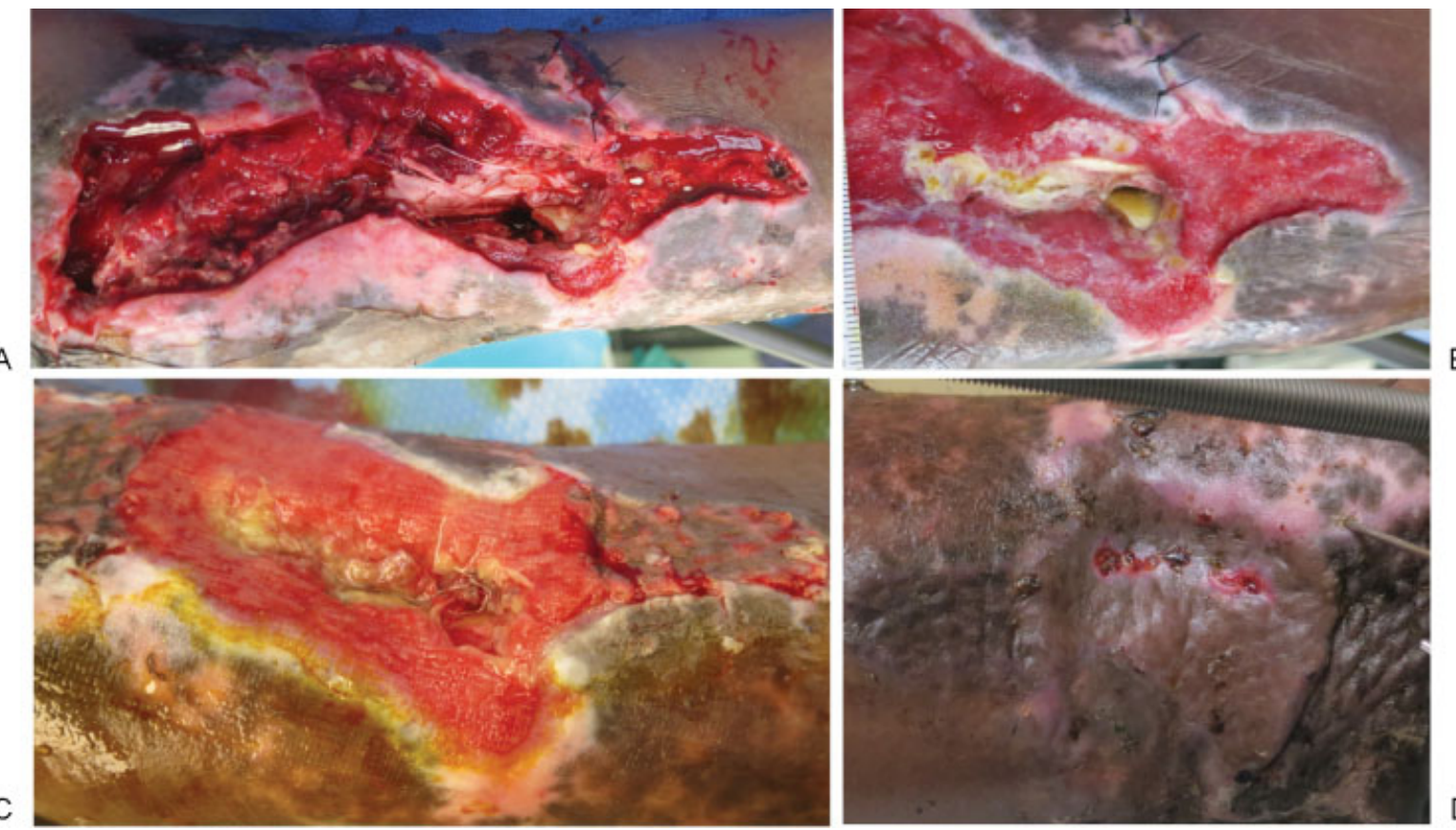

B

Fig. 3 A 47-year-old man presented with Gustilo 3B injury with exposed tibia and tendon following a motorcycle versus motor vehicle collision. (A) Wound in anterior lower extremity following debridement of devitalized tissue. (B) Wound following first dHACM treatment. (C) Wound after third dHACM application; STSG was applied at this time. (D) Stable closure 1 month following successful STSG, despite extensive bony manipulation for tibia re-reduction and conversion of external fixator to ring fixator. 
a local rotational flap which epidermolysed and ultimately closed with local wound care. The skin grafted portions of the wound remained stably closed at 2-month follow-up and the patient successfully resumed ambulation.

\section{Case III: Tibia and Tibialis Posterior Tendon}

A 47-year-old man presented with a Gustilo 3B injury involving $5 \mathrm{~cm}^{2}$ of exposed tibia and tibialis posterior tendon following a motorcycle collision ( - Fig. 3). Medical comorbidities included diabetes and peripheral arterial disease with single-vessel runoff on formal angiogram. Orthopedic surgery placed external fixation and negative pressure dressings, and consulted our service. We performed soft tissue debridement and three dHACM treatments, after which the wound demonstrated adequate granulation tissue STSG was successful. His closure remained stable at 1-month follow-up, despite requiring extensive subsequent bony manipulation due to loss of tibial reduction and conversion of the external fixator to a ring fixator.

\section{Discussion}

Extremity wounds with exposed critical structures, including bone and tendon, are a major burden on the American health care system with limited treatment options. Free flap reconstructions of lower extremity wounds have an increased failure rate in comparison to elective free flap procedures. Free flap reconstructions are limited due to technical expertise, access to care, and suitable patients. Although a larger sample size is needed to fully evaluate this novel treatment modality, our early experience suggests dHACM + STSG is a viable, low-cost alternative to free flap reconstruction. We have successfully applied this technique to six of seven patients. Additional advantages to this modality include a potential decreased time of hospital stay, avoiding immobility for the patient, and need for intensive care unit monitoring, leading to decreased need of ancillary services including nursing and physical therapy. Future studies will include a randomized controlled trial, and will be aimed at optimizing patient selection, timing of treatment, and analyzing the cost utility of dHACM and STSG in comparison to free flap reconstruction in addition to understanding the biological response within the wounds.

\section{References}

1 Colohan S, Saint-Cyr M. Management of lower extremity trauma. In: Neligan PC, ed. Plastic Surgery. 3rd ed. New York, NY: Elsevier; 2013:63-91

2 Hong S. Reconstructive surgery: lower extremity coverage. In: Neligan PC, ed. Plastic Surgery. 3rd ed. New York, NY: Elsevier; 2013:127-150

3 Khouri RK, Cooley BC, Kunselman AR, et al. A prospective study of microvascular free-flap surgery and outcome. Plast Reconstr Surg 1998;102(3):711-721

4 Gruss JS, Jirsch DW. Human amniotic membrane: a versatile wound dressing. Can Med Assoc J 1978;118(10):1237-1246

5 Zelen CM, Serena TE, Fetterolf DE. Dehydrated human amnion/ chorion membrane allografts in patients with chronic diabetic foot ulcers: a long-term follow-up study. Wound Med 2014:1-4

6 Serena TE, Carter MJ, Le LT, Sabo MJ, DiMarco DT; EpiFix VLU Study Group. A multicenter, randomized, controlled clinical trial evaluating the use of dehydrated human amnion/ chorion membrane allografts and multilayer compression therapy vs. multilayer compression therapy alone in the treatment of venous leg ulcers. Wound Repair Regen 2014;22(6): 688-693 